\title{
Evaluación de los estilos educativos familiares en la ciudad de Cuenca
}

\section{Palacios Madero M., Villavicencio Alvarado F., Mora Oleas C.}

Proyecto Estilos de Educación Familiar en la Ciudad de Cuenca, Facultad de Psicología, Universidad de Cuenca, Av. 12 de Abril s/n, Cuenca, Ecuador.

Autor para correspondencia: maria.palaciosm@ucuenca.edu.ec

Fecha de recepción: 16 de junio de 2015 - Fecha de aceptación: 31 de octubre de 2015

\section{RESUMEN}

Baumrind, psicóloga clínica del siglo 20, reconocida por su investigación sobre estilos de crianza distingue tres estilos de la educación familiar. Sustentado en este enfoque se inició un estudio para identificar el estilo educativo de los padres y las madres de la ciudad de Cuenca, Ecuador, y para la caracterización de las estrategias educativas que aplican. La población de estudio, fue seleccionada aleatoriamente y estuvo integrada por 445 padres/madres de niños de 6 a 7 años de edad que asistieron al segundo año de la educación básica de 19 escuelas urbanas: públicas y privadas. El análisis factorial distinguió los tres estilos educativos: autoritario, democrático y permisivo, planteados en el cuestionario. Del análisis cluster se deduce que en las familias cuencanas: (i) predominan padres/madres con un estilo educativo "no definido"; (ii) los padres/madres alternan estrategias educativas de los tres estilos, con una tendencia hacia prácticas educativas democráticas; (iii) no existe un estilo educativo dominante de acuerdo a la teoría de Baumrind; (iv) en consecuencia, existe una amplia oportunidad de investigar las tendencias educativas de los padres/madres. Sobre la base de estas conclusiones se recomienda la implementación de programas de educación parental para mejorar la crianza y el desarrollo de los hijos.

Palabras clave: Estilos educativos, padre/madre, familia.

\begin{abstract}
According to Baumrind, a 20th century clinical and developmental psychologist well known for her research on parenting styles, a distinction can be made between three styles of family education. Based on her work a project was initiated to characterize the educational style of the parents and single mothers of the city of Cuenca, Ecuador, and to unravel and characterize the educational strategies they apply. The study population consisted of 445 randomly selected parents of 6-7 year old children attending the second year of basic education at one of the 19 public and private urban schools. Factor analysis indicated that the education styles of the families can be classified in the three educational style classes defined by the classical theory, respectively the authoritarian, the democratic and the permissive. Additionally, results of the cluster analysis revealed that: (i) the undefined educational style predominates; (ii) parents/mothers alternate educational strategies of the three styles, with a trend toward democratic educational practices; (iii) no dominant educational style is applied according to Baumrind's theory; and (iv) ample opportunity exists to investigate the educational trends of parents/mothers. Based on these findings the implementation of parent education programs is recommended to improve the nurturing and development of children.
\end{abstract}

Keywords: Educational styles, father/mother, family. 


\section{INTRODUCCIÓN}

En el discurso social, existen distintas concepciones de la familia, unas afines al clima de afecto y apoyo que se brinda para un adecuado desarrollo psicológico de sus miembros, otras asociadas a la supervivencia, al cuidado, a la protección que brindan los padres para un sano crecimiento de sus hijos. Es decir, la familia es el primer escenario para el desarrollo físico, psicológico del desarrollo humano. Según, Ceballos \& Rodrigo (1988: 227), la familia es un contexto de desarrollo esencial para el niño, ya que le proporciona un marco ideal para socializarse, esto es, le prepara para lograr su adaptación a la sociedad a través del aprendizaje de valores, normas, comportamientos y en el proceso de socialización donde cobra especial relevancia la interacciones entre los miembros de la familia. Para Palacios \& Rodrigo (1998: 33) en la familia se comparten un proyecto vital de existencia en común que se quiere duradero, en el que se generan fuertes sentimientos de pertenencia a dicho grupo, existe un compromiso personal entre sus miembros y se establecen intensas relaciones de intimidad, reciprocidad y dependencia. En este sentido las relaciones interpersonales es uno de los rasgos esenciales en la familia, vinculadas a un contexto más amplio de la comunicación y la conducta. Por otra parte, la familia se convierte en un importante escenario para el aprendizaje, donde se enmarcan las estrategias y mecanismos de socialización y educación de los hijos. La función de socialización es sin lugar a dudas una de las funciones más ampliamente reconocidas de la familia. La socialización suele definirse como el proceso mediante el cual las personas adquirimos los valores, creencias, normas y formas de conducta apropiados en la sociedad a la pertenecemos (Musitu \& Cava, 2001).

Lógicamente, estos aspectos de la socialización familiar no son universales, sino que se encuentran relacionados con el contexto socio-cultural en el que se integra la familia. Es así que en el Ecuador, las presiones económicas sobre las familias, así como las transformaciones sociales han modificado su composición. Como consecuencia, las estructuras y arreglos que debe adoptar la organización familiar para garantizar su reproducción y supervivencia, no siempre permiten que los padres puedan cumplir con los roles o funciones de socialización encargados (Observatorio de los Derechos de la Niñez y la Adolescencia, 2010). Esto conlleva a que la paternidad y la maternidad se enfrente a problemas como el mantener la autoridad en una sociedad que da más espacio a los derechos de los niños y adolescentes; estar cerca afectivamente de los hijos e hijas cuando se está más tiempo fuera del hogar; evitar p.ej. estilos de crianza contradictorios entre los miembros de la pareja.

A pesar de la importancia vital que cumple la familia en el desarrollo social y emocional de los niños/as, se han realizado pocas investigaciones que proporcionen información sobre los estilos de educación familiar de los padres/madres de la ciudad de Cuenca. Este vacío de conocimiento local en el área de la socialización familiar, implica plantear investigaciones que nos permita entender las estrategias educativas parentales que configuran los estilos educativos y sus consecuencias en el desarrollo y ajuste social, emocional y conductual de los hijos. En esta línea, se presentan definiciones de estilo educativo similares con un significado práctico. Así, un estilo educativo tanto "representa la forma de actuar de los adultos respecto a los niños ante situaciones cotidianas, toma de decisiones o la solución de problemas" (Torio \& Rodríguez, 2008: 153), como los "esquemas prácticos que reducen las múltiples y minuciosas prácticas educativas paternas a unas pocas dimensiones, que, cruzadas entre sí en diferentes combinaciones dan lugar a diversos tipos habituales de educación familiar" (Coloma, 1993: 48).

En el ámbito de la socialización familiar, (Izzedin \& Pachajoa, 2009) indican que la crianza implica tres procesos psicosociales: las pautas de crianza, las prácticas de crianza y las creencias acerca de la crianza. Por un lado, las pautas se relacionan con la normatividad que siguen los padres frente a los comportamientos de los hijos y son portadoras de normas sociales. Las prácticas de crianza se ubican en el contexto de las relaciones entre los miembros de la familia, un conjunto de acciones, comportamientos aprendidos de los padres, ya sea a partir de su propia educación como por imitación y se exponen para guiar las conductas de los hijos. Además, las creencias hacen referencia al conocimiento acerca de cómo se debe criar a un niño, a las explicaciones que brindan los padres sobre la forma como encausan las acciones de sus hijos. Los procesos psicosociales de la crianza descritas en forma separada, se integran en un constructo que agrupa las prácticas educativas que 
habitualmente los padres utilizan en la relación con los hijos y que en el lenguaje cotidiano se llama Estilos de Educación Familiar.

En la investigación de la socialización familiar, uno de los modelos más elaborados es el de Baumrind (1967 y 1971). En estos estudios, se considera la interrelación entre las tres variables paternas básicas: control, comunicación y afecto. De la combinación de estas dimensiones, Baumrind pretende descubrir si determinados estilos educativos se correlacionan con el desarrollo de cualidades y características del niño. En cuanto a la metodología, en el primer estudio realizado en 1967, Baumrind dividió a 134 niños, de tres años de edad, n tres tipos de estructura personal según su conducta: a) los niños de la estructura (i) eran los más competentes, contentos e independientes, confiados en sí mismos y mostraban conductas exploratorias; b) los niños de la estructura (ii) eran medianamente confiados y capaces de controlarse a sí mismos y, en cierto modo, inseguros y temerosos; y c) los niños de la estructura (iii) se manifestaban inmaduros y dependientes, con menos capacidad de control y confianza en sí mismos. Seguidamente, se correlacionó la estructura de la personalidad con los métodos de crianza en la familia y se encontró; a) los padres del grupo (i), especialmente las madres ejercían un control firme, exigencia de ciertos niveles de madurez y buena comunicación con los hijos (denominados autoritativo parental); b) los del grupo (ii) eran menos cuidadosos con los hijos (denominados padres autoritarios); y c) los padres del grupo (iii) eran afectuosos y atentos, pero ejercían poco control y escasas demandas de madurez sobre sus hijos (denominados padres permisivos). En 1971, Baumrind, estudio la conducta de la misma muestra cuando los niños tenían 8/9 años y observó que los niños de familias democráticas tenían elevadas competencias sociales y cognitivas; los de progenitores autoritarios se situaban en un nivel medio y los niños de padres permisivos tenían niveles más bajos. Raya (2008) indica que este modelo determinaría profundamente el pensamiento posterior sobre los estilos parentales, que sin duda ha sido la más influyente en el ámbito de la psicología educativa.

Jiménez \& Guevara (2010) indican que a partir de la tipología planteada por Baumrind, Maccoby \& Martin (1983) redefinen los estilos educativos en función de las dimensiones subyacentes: control y afecto. De la combinación de estas dos dimensiones surgen los cuatro estilos siguientes: democrático, autoritario, indulgente y negligente. El estilo permisivo de Baumrind es desglosado en permisivo indulgente y permisivo negligente.

Los estilos educativos tienen una gran repercusión y consecuencias evolutivas que no se circunscriben sólo a la etapa infantil, sino que se prolongan a lo largo de la vida. No es sorprendente que en los últimos años a nivel internacional muchos estudios sobre la crianza de los hijos de la familia se han realizado, lo que dio lugar a un amplio volumen de artículos de investigación interesantes. Torio et al. (2008) describen investigaciones que han analizado la relación entre las tendencias de comportamiento de los padres y el desarrollo de los hijos. Los autores más importantes se resumen en lo siguiente:

- Estilos educativos y el éxito o competencia académica de los hijos: Chao (1994), Ballantine (2001), Burchinal et al. (2002), Kim \& Rohner (2002), Pérez et al. (2001), Balzano (2002), Peralbo \& Fernández (2003), Hernando et al. (2012), Belarcazar \& Delgado (2013).

- La dimensión de afecto por parte de los padres con el bienestar psicológico y con un nivel óptimo de autoestima en los hijos: Mestre \& Frías (1997), García et al. (2002), Kim \& Chung (2003), Alonso García \& Román Sánchez (2003), Muñoz (2005), Olivia \& Parra (2004), Olivia et al. (2008), Clereci \& García (2010), Pérez (2012).

- Estilo disciplinar de los padres en la configuración de valores de sus hijos: Hoffman (1975a, 1975b), Grusec \& Goodnow (1994), Molpereces et al. (1994), Molpereces et al. (2000), Ortega \& Mínguez (2003), Elzo (2010), Mínguez (2012).

- Prácticas educativas paternas y la competencia psicosocial de los hijos: Lamborn et al. (1991), Musitu et al. (1996), Martínez \& Fuertes (1999), Aunola et al. (2000), Herrera et al. (2001), García et al. (2002), Oliva \& Parra (2004), Ramírez (2005) Oliva et al. (2008), García \& Gracía (2010).

- Estilos de vida de adolescentes en riesgo y la calidad de las relaciones padres-hijos: Corvo \& Williams (2000), Rodrigo et al. (2004), Orte Socias (2005, 2006), Estébez et al. (2007), Raya 
(2008), Musitu et al. (2009), Álvarez (2010), Gómez et al. (2014), García-Linares et al. (2014).

- Un tema de investigación que merece ser destacado por las implicaciones en el desarrollo de los hijos es la conducta de los padres asociada a la consistencia-inconsistencia interparental: Arce et al. (2003), Gracia et al. (2004); Rodríguez et al. (2009), García el al. (2014). Independientemente del estilo predominante, las inconsistencias resultan perjudiciales por considerarse como factor que afecta posibles: cogniciones distorsionadas, emociones disfuncionales y conductas problema en los niños.

En comparación con la literatura internacional, la contribución del Ecuador para el campo es muy limitada, y consta de Guerra (2009), Buestán \& Mendieta (2012) y Coronel \& Sánchez (2013) quienes describen la relación de las prácticas educativas con el desarrollo cognitivo, desarrollo integral y cuidado de los niños de 0 a 5 años, respectivamente.

Si bien, todos los estudios mencionados hacen hincapié en la influencia de la conducta parental sobre los hijos, en la cotidianidad familiar, las estrategias educativas paternas no siempre responden a un solo estilo educativo, sus prácticas son diversas. Estudios en este ámbito se enfocan a analizar posibles inconsistencias entre los estilos de ambos progenitores, planteando la hipótesis general que las inconsistencias paternas resultan perjudiciales para el desarrollo de los hijos. Berkien et al. (2012).

De esta forma tanto la variabilidad de situaciones y respuestas educativas, como posibles inconsistencias parentales hacen difícil encasillar a las familias en una u otra tipología, porque la manera como se comporta un padre respecto a un niño en particular depende de la edad del niño, de su apariencia física, de su conducta habitual, de su conducta pasada, su inteligencia y su estado de salud. Los padres confeccionan su estilo educativo a medida de cada niño, la educación no es algo que los padres hagan a los hijos, sino algo que los padres e hijos hacen conjuntamente (Rich Harris, 2002: 53). Por tanto, cuando se habla de estilos de prácticas educativas parentales, se hace referencia a tendencias globales de comportamiento, ya que con ello no se pretende señalar que los padres hagan lo mismo con todos los hijos ni en todas las situaciones. En esta línea, Rodrigo \& Palacios (1998) postulan que las prácticas educativas de los padres pueden estar determinadas por una serie de factores que se dividen en tres grupos:

- Un primer grupo relacionado con el niño: edad, sexo, orden de nacimiento, número de hermanos y características de personalidad. Los atributos del niño: por ejemplo demandantes y emocionalmente negativos, resultan más difíciles de manejar.

- Un segundo grupo relativo a los padres: sexo, experiencia previa como hijos y como padres, características de personalidad, nivel educativo, ideas acerca del proceso evolutivo y la educación y expectativas de logro que tienen puestas en sus hijos. En cuanto al estilo educativo de los padres por el sexo, muchos hijos siguen viendo a los padres como disciplinadores y a las madres como comprensivas y aliadas en la búsqueda de espacios de autonomía (Meil, 2006). Pons-Salvador et al. (2005) identificaron factores asociados a los cambios que afecta negativamente a la parentalidad, como los antecedentes de castigo en la madre, la baja satisfacción materna y la percepción negativa del bebé entre otros, que se convierten en factores de riesgo para maltrato y alteraciones emocionales. A criterio de Millon (2000) el tipo de vínculo afectivo y el manejo de la norma, temperamento y actitudes que tienen los padres para con los hijos pasan a ser generadores de estilos comportamentales y actitudinales adecuados.

- Un tercer grupo relacionado con la situación en la que se lleva a cabo la interacción: características físicas de la vivienda, contexto histórico, situación en que se lleva la interacción e influencia de la estructura familiar en las características de las prácticas de crianza de los padres. En la conducta de los padres cuando se relacionan con los hijos tiene una influencia relativa el número de adultos del contexto, el número de niños, la educación de la madre, el ingreso familiar. Investigaciones revelan la importancia del nivel de estudios y profesional de los padres, metas educativas de los padres y de su propia historia evolutiva, así como de las características de los hijos y de la configuración familiar entre otros muchos factores (Ceballos \& Rodrigo, 1998; Grusec, 2002; Palacios, 1999; Palacios \& Moreno, 1995). 
Según Meil (2006) la cultura actual ha dado origen a una mayor individualización, autonomía y protagonismo de las personas. Así las aspiraciones vitales de las mujeres han dejado de definirse en la sociedad actual sólo en el ámbito familiar como una vida orientada al servicio de la familia, para afirmarse en su derecho de tener también una carrera profesional propia; como consecuencia los roles conyugales, el reparto de responsabilidades, derechos y obligaciones han modificado las prácticas de crianza y los estilos educativos. En definitiva, el estilo parental puede presentar variaciones en función de determinados factores contextuales y personales tanto de padres e hijos, a nivel personal, como del contexto familiar y social en general. No obstante, más allá de que se determine la existencia o no de un estilo predominante, es necesario visualizar las dimensiones de afecto, comunicación, control en una interacción de calidad (Aroca \& Cánovas, 2012).

Desde una concepción tipológica de los estilos educativos, esta investigación, se plantea como objetivo describir las estrategias educativas que presentan los padres y las madres en cada estilo educativo al momento de educar a sus hijos en el contexto de las familias cuencanas.

\section{MATERIALES Y METODO}

El estudio fue parte del proyecto de investigación: "Estilos de Educación Familiar en la Ciudad de Cuenca“, proyecto ganador del XII concurso convocado por el Departamento de Investigación de la Universidad de Cuenca. (2013-2014). Se trató de un estudio cuantitativo, descriptivo de carácter transversal.

\subsection{Muestreo de población}

A partir de la base de datos proporcionada por la Dirección de Planificación de la Coordinación de Educación Zona 6, se determinó una población de 147 instituciones educativas fiscales y particulares correspondientes al distrito 1 y 2 del cantón Cuenca. De las cuales se seleccionaron aleatoriamente 12 escuelas fiscales y 7 particulares. Participaron en el estudio: 445 padres y madres de familia de los niños/as del segundo año de educación básica (6-7 años). La muestra está calculada con un nivel de confianza de $95 \%$ y un error muestral de $5 \%$. Tabla 1 presenta las características sociodemográficas de la muestra.

Tabla 1 Características sociodemográficas de la muestra.

\begin{tabular}{llrr}
\hline Variable & & \multicolumn{1}{c}{ Padres } & \multicolumn{1}{c}{ Madres } \\
\hline \multirow{4}{*}{ Edad } & Media & 34.52 años & 32.38 años \\
& DE & 6.26 años & 6.17 años \\
& Mínima & 21 años & 21 años \\
& Máxima & 50 años & 50 años \\
\hline \multirow{4}{*}{ Ocupaciones } & $43.3 \%$ & $43.5 \%$ \\
& Profesionales, científicas y técnicas & $17.9 \%$ & $15.7 \%$ \\
laboral & Quehaceres domésticos & & $25.8 \%$ \\
& Transporte y almacenamiento & $13.2 \%$ & $0.4 \%$ \\
& Comercio & $10.8 \%$ & $6.5 \%$ \\
& Ninguna & $2.7 \%$ & $2.7 \%$ \\
& No contesta & $12.1 \%$ & $5.4 \%$ \\
\hline \multirow{5}{*}{ Nivel de } & Superior completo & $22 \%$ & $30 \%$ \\
educación & Superior incompleto & $17 \%$ & $17 \%$ \\
& Bachillerato & $28 \%$ & $26 \%$ \\
& Educación básica & $26 \%$ & $25 \%$ \\
& Ninguno & $2 \%$ & $2 \%$ \\
& No contesta & $5 \%$ & $0 \%$ \\
\hline
\end{tabular}


Se encontró una relación significativa, 0.57 , entre el nivel educativo de la madre con respecto al del padre. El mayor porcentaje de padres con educación superior completa se concentra en escuelas particulares con un 39.3 y $50 \%$, frente a un 14.1 y $19.4 \%$ en las escuelas fiscales. En cuanto a la situación laboral, en las escuelas fiscales, es donde se encuentran la mayor cantidad de padres con empleo y a tiempo completo, en un $93.6 \%$ frente a un $88.5 \%$ de padres que tienen hijos en las escuelas privadas. En las escuelas fiscales existe un $7.6 \%$ de madres desempleadas y en un $47.6 \%$ dedicadas a los quehaceres domésticos.

En la Tabla 2 la nuclearización de la familia queda definida por el porcentaje de padres casados y de unión de hecho (85\%), en general estas familias tienen entre 1-2 hijos (41 y 24\% respectivamente). Otras investigaciones en el medio reportan valores cercanos al $70 \%$ de niños que viven con sus padres y un 60\% de familias con 1 o más hijos (Shephard et al., 2015). Con lo cual se confirma que la estructura familiar Cuencana es nuclear y se caracteriza por la presencia del subsistema fraterno en más del $60 \%$ de familias. Por otro lado, el $23 \%$ afirma vivir por lo menos con uno de los abuelos del niño. Teniendo como resultado que la mayoría de los hogares están conformados entre 4-5 personas (31.7 y $21.9 \%$ respectivamente).

Tabla 2. Estructura familiar y estado conyugal.

\begin{tabular}{lr}
\hline Estructura familiar & \\
\hline Nuclear & $65 \%$ \\
Trigeneracional & $14 \%$ \\
Monoparental & $15 \%$ \\
No contesta & $6 \%$ \\
\hline
\end{tabular}

\begin{tabular}{lr}
\hline Estado conyugal padres encuestados \\
\hline Casados & $68.4 \%$ \\
Unión de hecho & $16.6 \%$ \\
Solteros (madres) & $7 \%$ \\
Separados & $4 \%$ \\
Divorciados & $4 \%$ \\
\hline
\end{tabular}

Se debe destacar que el $82 \%$ de padres y el $99 \%$ de madres, al momento de la aplicación del cuestionario, afirman vivir en el núcleo familiar, esto indica que las madres tienen una presencia permanente dentro del núcleo familiar, a diferencia de los padres que tienen mayor movilidad familiar. Estos datos explican la participación de los padres en las respuestas al cuestionario, este fue contestado por las madres en un $48 \%$ seguido de la respuesta conjunta de padre y madre en un $41 \%$, lo que implica que a pesar que tradicionalmente existe una alto involucramiento de las madres en las actividades escolares de sus hijos, se visualiza una tendencia de una mayor colaboración de ambos padres.

\subsection{Recolección de información}

El instrumento para la recolección de la información fue el cuestionario "Estudio socioeducativo de hábitos y tendencias de comportamiento en familias con niños de segundo año de Educación Básica" Torío (2003). Consta de cinco partes: datos sobre la unidad familiar, organización de la vida familiar, recursos sociales y/o comunitarios, tendencias actitudinales educativas y percepción social. Para responder a los objetivos formulados, el análisis de los datos se concentró en los trece ítems de la pregunta 25 del bloque tendencias actitudinales educativas que se refieren a las estrategias que utilizan habitualmente los padres y madres cuando se relacionan con sus hijo/as. Los encuestados debían expresar su acuerdo, sentimiento y aprecio utilizando la escala de Likert, cuyas respuestas oscilaban entre 1 (muy de acuerdo), 2 (en desacuerdo), 3 (de acuerdo), 4 (muy en desacuerdo). Dicha escala permite establecer los estilos educativos de los padres y madres encuestados, de acuerdo a la tipología clásica de Baumrind (Autoritativo, Permisivo y Autoritario). Al igual que en el estudio de Torio (2003), por cada bloque de preguntas en el cuestionario se aplica el análisis factorial.

Para la recolección de la información, con la autorización a la Coordinación Zonal 6 de Educación se ingresó a las escuelas fiscales y particulares, se coordinó con los directores y docentes las fechas de reunión de entrega de certificado de calificaciones del primer quimestre del curso académico para explicar a los padres /madres de familia sobre la estructura del cuestionario y la importancia de las respuestas para la investigación. Los padres y madres llevaron el instrumento y la hoja de asentimiento informado en un sobre cerrado junto con la hoja explicativa. Los cuestionarios 
resueltos fueron recogidos por la profesora de cada grado al que asistían los hijos de los padres participantes en las 19 escuelas.

\subsection{Análisis de datos}

El procesamiento de los datos fue realizado con el programa estadístico SPSS versión 20. Para la interpretación y análisis de los resultados se utilizó como medida estadística el análisis factorial mediante el método de componentes principales para analizar la interacción entre las variables de los estilos educativos. Se apreció una buena adecuación de los datos a través del estadístico Test de Kaiser-Meyer-Olbin (KMO) que mide la idoneidad de los datos, presentando un valor de 0.720. Para probar la homogeneidad de varianzas, la prueba de Esfericidad de Bartlett $\left(x^{2}=573.070\right.$, Sig $\left.=0.00\right)$ presentó los valores adecuados para la utilización de un análisis factorial. Con el objetivo que las variables que integran los estilos educativos estén mejor definidas dentro de los componentes se realizó una rotación varimax de los factores, el mismo que garantiza la no correlación entre los componentes.

Con la finalidad de encontrar grupos de padres/madres de familia con estilos educativos definidos, se utilizó el análisis de cluster, para clasificar la muestra en distintos utilizando variables que midan similitudes entre los individuos. El método de conglomeración empleado es la vinculación intergrupos mediante la distancia Euclídea al cuadrado.

\section{RESULTADOS Y DISCUSIÓN}

Los tres factores que corresponden a los tres estilos educativos de acuerdo a la teoría de Baumrind $(1967,1971)$, son interpretados a continuación. La Tabla 3 presenta los resultados relacionados con el estilo democrático, la Tabla 2 la varianza, descripción y saturación del factor que caracteriza el estilo permisivo y la Tabla 3 finalmente las misma información sobre el estilo autoritario.

Tabla 3. Estilo democrático: varianza explicada, descripción y saturación del factor.

\begin{tabular}{lc}
\hline Estilo DEMOCRÁTICO & Porcentaje de varianza explicada: $22.27 \%$ \\
\hline Descripción & Saturación \\
\hline $\begin{array}{l}\text { En mi opinión, es normal que mis hijos cometan errores mientras están } \\
\text { aprendiendo. }\end{array}$ & .745 \\
$\begin{array}{l}\text { Me agrada que mis hijos tengan iniciativa para hacer cosas, aunque } \\
\text { cometan errores. }\end{array}$ & .739 \\
$\begin{array}{l}\text { Los niños aprenden mejor a través del juego. } \\
\text { El diálogo es el mejor sistema para conseguir que los niños }\end{array}$ & .662 \\
comprendan. & .458 \\
\hline
\end{tabular}

Se encuentran en este factor 4 elementos, Tabla 3, estas afirmaciones revelan que en el estilo democrático, como primer elemento configurador, (0.745) se ubica como estrategia educativa la autonomía, la iniciativa, "aceptación de errores mientras está aprendiendo". Esto evidencia que los padres democráticos otorgan autonomía a los hijos en el desarrollo de competencias sociales partiendo de los derechos y deberes de los hijos. El último elemento configurador de este estilo, es "el diálogo como mejor sistema para que los niños comprendan" (0.458), revelando que los padres, en menor grado utiliza esta estrategia educativa en la crianza de los hijos.

Las afirmaciones que configuran el estilo permisivo, Tabla 4, son estrategias educativas centradas en la libertad que se otorga a los hijos. El primer elemento configurador, (0.772) "creo que los padres tenemos que dejar a los hijos a su libertad para que aprendan por sí mismos", "creo que la vida es la mejor escuela, sin que sea necesario estar dando constantemente consejos a los hijos" con (0.561) y "me molesta cuando alguno de mis hijos me pida ayuda para hacer algo; creo que debería hacerlo solo" (0.526) evidencian poca exigencia paterna, no se acentúa la autoridad, pues estos padres 
no establecen normas estrictas en la distribución de tareas en el hogar, ni en los horarios para comer, dormir, permisos. Los padres permisivos tienen mayor tolerancia a los errores de los hijos, dejan que los hijos hagan lo que quieran sin corregirles ni orientarles evitando los castigos. El estilo permisivo o no restrictivo refleja una relación padre e hijo no directiva basada en el no control parental y la flexibilidad (Baumrind, 1997), citado por (Henao et al., 2007), esta práctica educativa de los padres podría generar confusión en los hijos pues las normas y las jerarquías son necesarias para el desarrollo saludable de los niños, al desarrollar autonomía, seguridad en sí mismos, desarrollo del autocontrol.

Tabla 4. Estilo permisivo: varianza explicada, descripción y saturación del factor.

\begin{tabular}{lc}
\hline Estilo PERMISIVO & Porcentaje de varianza explicada: $15.91 \%$ \\
\hline Descripción & Saturación \\
\hline $\begin{array}{l}\text { Creo que los padres tenemos que dejar a los hijos a "su libertad" para } \\
\text { que aprendan por sí mismos. }\end{array}$ & .772 \\
$\begin{array}{l}\text { Creo que la vida es la mejor escuela, sin que sea necesario estar dando } \\
\text { constantemente consejos a los hijos. }\end{array}$ & .561 \\
$\begin{array}{l}\text { Me molesta que alguno de mis hijos me pida ayuda para hacer algo; } \\
\text { creo que debería hacerlo solo/a. }\end{array}$ & .526 \\
\hline
\end{tabular}

Tabla 5. Estilo autoritario: varianza explicada, descripción y saturación del factor.

\begin{tabular}{lc}
\hline Estilo AUTORITARIO & Porcentaje de varianza explicada: $10.07 \%$ \\
\hline Descripción & Saturación \\
\hline $\begin{array}{l}\text { La clave para educar bien a los hijos consiste, esencialmente, en } \\
\text { castigarlos cada vez que se portan mal. }\end{array}$ & .741 \\
$\begin{array}{l}\text { Los padres tenemos que utilizar frecuentemente los castigos para } \\
\text { prevenir problemas futuros. }\end{array}$ & .710 \\
Los niños deben respetar siempre a los padres por el hecho de serlo. & .463 \\
\hline
\end{tabular}

Los tres componentes que configuran el estilo autoritario, Tabla 5, se refieren al mérito de la obediencia, respeto a la autoridad, y a la aplicación del castigo a los hijos. Siendo el castigo el primer elemento configurador y máximo definidor de este factor (0.741). Esta postura autoritaria tiene la singularidad de acentuar la jerarquía de su rol de padres, la rigidez de la disciplina y la obediencia como mecanismos para educar a sus hijos, desvirtuando el diálogo y la comunicación entre padres e hijos; esta idea les lleva a no valorar los deseos y no dar respuesta a las necesidades emocionales de los hijos. Padres y madres que utilizan este estilo educativo muestran niveles bajos de afecto/comunicación y niveles altos de exigencia y control. Este modelo ha sido uno de los más discutidos tanto en el discurso social como en la literatura, quizá porque se ha presentado con más frecuencia en las prácticas educativas, generando en los hijos baja autoestima, baja percepción de control de sus propias situaciones, sentimientos de miedo, desagrado, rencor, rebeldía, rechazo a quienes representan a los padres/madres que representan ese modelo.

Una vez obtenidos los factores resultantes que corresponde a los estilos educativos, se utilizaron las puntuaciones factoriales para una reagrupación de la muestra mediante el análisis cluster exploratorio. Al analizar el dendograma se visualizó que la muestra se agrupaba alrededor de 4 clusters.

La Tabla 6 presenta la media y la desviación estándar de los puntajes factoriales para los grupos identificados en cada uno de los estilos educativos, con el fin de conocer en qué estilo se ubica cada uno de los cuatro grupos. El primer grupo "no definido" está conformado por la mayoría de la muestra, 434 padres/madres, un segundo grupo conformado por 3 padres se distingue por tener un estilo permisivo, el valor de la media en este estilo es positivo, de igual forma un tercer grupo formado por 3 padres que responden al estilo autoritario; finalmente el grupo 4 con 2 padres responden a un estilo no reconocido, ya que las puntuaciones para la media son negativas. 
Tabla 6. Análisis cluster y estilos educativos, media y DT de los puntajes factoriales.

\begin{tabular}{|c|c|c|c|c|c|c|c|c|}
\hline \multirow{3}{*}{$\begin{array}{l}\text { Estilos } \\
\text { educativos }\end{array}$} & \multicolumn{8}{|c|}{ Descripción de grupos } \\
\hline & \multicolumn{2}{|c|}{$\begin{array}{c}\text { 1. No definido } \\
\mathrm{n}=343\end{array}$} & \multicolumn{2}{|c|}{$\begin{array}{l}\text { 2. Permisivo } \\
\mathrm{n}=3\end{array}$} & \multicolumn{2}{|c|}{$\begin{array}{c}\text { 3. Autoritario } \\
n=3\end{array}$} & \multicolumn{2}{|c|}{$\begin{array}{l}\text { 4. No reconocido } \\
\qquad \mathrm{n}=2\end{array}$} \\
\hline & Media & DT & media & DT & Media & DT & media & DT \\
\hline Democrático & 0.05 & 0.93 & -1.36 & 0.45 & -2.7 & 0.68 & -4.5 & 0.02 \\
\hline Permisivo & -0.07 & 0.98 & 2.66 & 0.63 & -0.429 & 0.42 & -1.73 & 0.12 \\
\hline Autoritario & 0.008 & 0.96 & -1.67 & 0.54 & 1.43 & 0.21 & -1.34 & 1.22 \\
\hline
\end{tabular}

El análisis bivariado entre los grupos identificados a través del análisis cluster y los puntajes directos correspondientes a los estilos educativos, permitió determinar las particularidades del estilo no definido. Para el cual se registró una media de 3.46 cercana al máximo valor esperado en la escala y medias de 2.34 y 2.37 para indicadores autoritarios y permisivos, puntajes ligeramente inferiores a la media de la escala. En consecuencia el estilo no definido se caracteriza por integrar los tres estilos educativos, con una marcada tendencia hacia el estilo democrático que coexiste con tendencias autoritarias y permisivas en menor grado (Tabla 7).

Tabla 7. Distribución de la muestra según análisis cluster y estilos educativos, puntajes directos.

\begin{tabular}{|c|c|c|c|c|c|c|c|c|}
\hline \multirow{3}{*}{$\begin{array}{c}\text { Estilos } \\
\text { educativos }\end{array}$} & \multicolumn{8}{|c|}{ Descripción grupo } \\
\hline & \multicolumn{2}{|c|}{$\begin{array}{c}\text { 1. No definido } \\
\mathrm{n}=434\end{array}$} & \multicolumn{2}{|c|}{$\begin{array}{l}\text { 2. Permisivo } \\
\mathrm{n}=3\end{array}$} & \multicolumn{2}{|c|}{$\begin{array}{c}\text { 3. Autoritario } \\
n=3\end{array}$} & \multicolumn{2}{|c|}{$\begin{array}{l}\text { 4. No reconocido } \\
\qquad \mathrm{n}=2\end{array}$} \\
\hline & Media & $\mathrm{DE}$ & Media & $\mathrm{DE}$ & Media & $\mathrm{DE}$ & Media & $\mathrm{DE}$ \\
\hline Autoritario & 2.34 & 0.5 & 1.75 & 0 & 3 & 0.25 & 1.38 & 0.53 \\
\hline Democrático & 3.46 & 0.41 & 2.75 & 0.43 & 2.33 & 0.29 & 1.75 & 0 \\
\hline Permisivo & 2.37 & 0.43 & 2.6 & 0.92 & 2.47 & 0.42 & 1.7 & 0.14 \\
\hline
\end{tabular}

\section{CONCLUSIONES}

En relación con el estilo educativo que prevalece en las familias cuencanas, se deduce que del análisis de cluster se identifica 434 padres y madres de niños/as de segundo año de educación básica de las escuelas de la ciudad de Cuenca como "no definidos", porque no tienen puntuaciones medias que les permita ubicarse en un estilo educativo de acuerdo a la tipología de Baumrind; sin embargo, en la cotidianidad de la relación entre padres e hijos los padres manejan estrategias educativas utilitarias que al final responden a los tres estilos. Este grupo calificado como "no definido" está caracterizado por prácticas educativas con tendencias democráticas y escasas estrategias autoritarias y permisivas.

Es evidente que en el proceso educativo los padres/madres utilizan estrategias educativas, entendidas éstas, como un conjunto de conductas que los padres valoran como adecuadas para establecer normas y valores que favorezca la integración social de los hijos/as. Estas estrategias que emplean los padres, y que están basadas en las dimensiones de afecto - comunicación y control planteadas por Baumrind, son tendencias actitudinales, y no pueden ser concebidas en un sentido puro, más bien entenderlas como tendencias que nos acercan a la realidad de la familia cuencana.

Los resultados del presente estudio confirman los obtenidos en la investigación "Estudio socioeducativo de hábitos y tendencias de comportamiento en familias con niños de educación infantil y primaria en Asturias" España, de Torío et al. (2008a, 2008b) donde el 87.2\% de padres no tienen un estilo definido y el $12 \%$ si tienen un estilo definido que es el democrático. La autora indica que "no se identifican modelos puros, sino que se solapan, ya que los estilos educativos suelen ser mixtos y varían con el desarrollo del niño, no siendo estables a lo largo del tiempo". Concomitantemente en la investigación de Cuenca, Ecuador el 97.7\% (434 familias) no tienen un estilo definido y más bien 
combinan los tres estilos de crianza con una orientación democrática; y, un grupo muy reducido de familias con un estilo educativo definido, en este sentido, los datos no difirieren a la investigación realizada en España.

A partir del análisis factorial se destacan las siguientes tendencias de las estrategias educativas de los padres y madres de familia del cantón Cuenca:

- Una tendencia del comportamiento de los padres hacia un estilo democrático. Los padres/madres en la interacción con los hijos valoran la expresión de la autonomía, estimulan la iniciativa, valoran el juego y en menor grado, el diálogo en el desarrollo psicológico de los hijos. Se trata de una familia que da valor a la calidez, al afecto, al diálogo con un control de las normas, reglas claras y firmes en un clima de igualdad y democratización entre los miembros de la familia. Este estilo produce, en general efectos positivos en la socialización: desarrollo de competencias sociales, índices más altos de autoestima y bienestar psicológico, un nivel inferior de conflictos entre padres e hijos, entre otras consecuencias. Los niños de padres democráticos suelen ser interactivos y hábiles en sus relaciones con sus iguales, independientes y cariñosos (Izzedin \& Pachajoa, 2009) este tipo de crianza se encuentra en sintonía con la titularidad de derechos de los niños y adolescentes, es la crianza humanizada. Se puede afirmar que los estudios que analizan la relación entre los estilos educativos democráticos y consecuencias educativas en el desarrollo del niño coinciden en resaltar que este estilo caracterizado por el afecto, control, comunicación y exigencia de madurez está relacionado con los mejores resultados. (Alonso García \& Román Sánchez, 2003; Chang, 2003; Hernando et al., 2012). Estos resultados dejan ver la incidencia del impacto de los estilos educativos familiares en el desarrollo de los hijos.

- En los padres/madres encuestados, junto a esta tendencia democrática se constata una aceptación minoritaria a las prácticas autoritarias que valoran la obediencia como una virtud, así como la dedicación a las tareas marcadas, la tradición y la preservación del orden. Favorecen las medidas de castigo o de fuerza y están de acuerdo en mantener a los niños en un papel subordinado y en restringir su autonomía. Dedican muchos esfuerzos a influir, controlar y evaluar el comportamiento y actitudes de sus hijos de acuerdo con unos rígidos patrones preestablecidos. No facilitan el diálogo y, en ocasiones, rechazan a sus hijos/as como medida disciplinaria. El estilo autoritario es el que tiene repercusiones más negativas (Torío et al., 2008b: 158) sobre la socialización de los hijos, como la falta de autonomía personal y creatividad, menor competencia social o baja autoestima y genera niños descontentos, reservados, poco tenaces a la hora de perseguir metas y poco comunicativos y afectuosos y tienden a tener una pobre interiorización de valores morales. Sobre el área de autoridad familiar en relación con los comportamientos agresivos, son ilustrativos los trabajos de Musitu \& García (2004), Martínez \& Fuertes (1999), Agudelo (1993), Henao et al. (2007), Alonso García \& Román Sánchez (2005), Oliva et al. (2008), Sandoval et al. (2006).

- Finalmente los padres/madres encuestados, en relación con las estrategias educativas que configuran el estilo permisivo, de acuerdo al análisis factorial las pautas educativas están centradas en la concepción que tienen los padres en otorgar la libertad a los hijos para que aprendan por sí mismos, sin embargo en el análisis de cluster, este estilo tiene una puntuación debajo de la media -0.07. Los resultados obtenidos por (Perez, 2012), refuerzan la idea de que en las familias españolas, el estilo idóneo para la socialización parental es el indulgente, puesto que los hijos de estas familias siempre fueron equivalentes o superiores al estilo autoritativo. Los resultados de este estudio ratifican y extienden algunos estudios previos (Musitu \& García, 2004; García \& Gracia, 2010).

Padres y madres de las familias cuencanas, demuestran la utilización de estrategias educativas inconsistentes al momento de educar a los hijos. A criterio de Llanova \& Méndez (2012) uno de los errores en la educación familiar, es la inconsistencia que se caracteriza por la falta de estabilidad y coherencia en las estrategias de control, supervisión y disciplina empleadas en la educación de los hijos. En un estudio con adolescentes españoles, se ha encontrado relaciones significativas entre la inconsistencia y las manifestaciones emocionales de los hijos, particularmente, con la agresión y la depresión (Rodríguez et al., 2009). Un contexto de crianza consistente genera seguridad y permite que 
los hijos establezcan claras relaciones entre su propia conducta y las contingencias asociadas de la crianza familiar. De esta forma, se genera un adecuado contexto de aprendizaje y desarrollo que facilita la interiorización de normas y valores de los padres. A partir de lo señalado y los resultados de la investigación es evidente la necesidad de implementar programas de educación parental que potencien mayores niveles de comunicación, afecto y control para desarrollar en los hijos reflexión, autonomía, responsabilidad y bienestar emocional.

\section{AGRADECIMIENTOS}

El proyecto "Estilos de Educación Familiar en la Ciudad de Cuenca" agradece a las autoridades de la Universidad de Cuenca y al Director de la DIUC, Ing. Jaime Bojorque por el apoyo constante al equipo de investigación, lo que ha favorecido la formación de los miembros del equipo en la investigación científica y en la consecución de resultados que brinden una respuesta académica a las necesidades sociales de la familia cuencana. También reconoce y agradece el apoyo recibido por las autoridades y personal administrativo de la Facultad de Psicología. Gratitud a la factibilidad para la realización de la investigación fue posible gracias al apoyo teórico y metodológico de la Dra. Susana Torio de la Universidad de Oviedo (España) quien autorizó la aplicación del instrumento de investigación. De la misma manera nuestro reconocimiento a las autoridades de la Coordinación de Educación Zona 6, que autorizaron el ingreso a las instituciones educativas fiscales, a los directores y docentes de las escuelas de la ciudad de Cuenca por favorecer la aplicación y la recolección de la información.

\section{BIBLIOGRAFÍA}

Agudelo, R.E., 1999. Estilos educativos paternos: aproximación a su conocimiento. Universidad Pedagógica Nacional, Bogotá, Colombia. Revista Colombiana de Educación, 11, 1-12.

Alonso García, J., J. Román Sánchez, 2003. Educación Familar y autoconcepto en los niños pequeños. Madrid: Pirámide.

Alonso García, J., J.M. Román Sánchez, 2005. Prácticas educativas y autoestima, Psicothema, 17(1), 76-82.

Álvarez, M., 2010. Practicas educativas familiares, autoridad familiar incidencia en el comportamiento agresivo infantil. Revista Virtual Universidad Católica del Norte, (31), 253-273.

Arce, R., M. Novo, A. Carballal, 2003. Sensibilización acerca de las consecuencias negativas que provoca el conflicto interparental sobre los hijos. Revista Galego-Portuguesa de Psicología de la Educación, 10, 1138-2663.

Aroca, C., P. Cánovas, 2012. Los estilos educativos parentales desde los modelos interactivos y de construcción conjunta: revisión de las investigaciones. Teoría de la Educación, 24, 149-176.

Aunola, K., H. Stattin, J.E. Nurmi, 2000. Parenting styles and adolescents' achievement strategies. Journal of Adolescence, 23(2), 205-222.

Balzano, S., 2002. Las construcciones culturales sobre el éxito y el fracaso escolar y sus implicaciones sobre los modelos educativos en Argentina. Cultura y Educación, 3(14), 283-296.

Ballantine, J., 2001 Raising competent kids: the authoritative parenting style, Childhood Education, $78,46-47$.

Baumrind, D., 1967. Child care practices anteceding three patterns of preschool behavior. Child Development, 37(4), 887-907.

Baumrind, D., 1971. Current patterns of parental authority. Develomental Pyschology Monographs, 4(1, Part 2). 
Baumrind, D., 1997. The discipline encounter: Contemporary issues. Aggression and Violent Behavior, 2(4), 321-335.

Belalcazar, L., A. Delgado, 2013. Practicas educativas familiares en el desempeño escolar. Plumilla Educativa, 417-430.

Berkien, M., A. Louwerse, F. Verhulst, J. Van der Ende, 2012. Children perceptions of dissimilarity in parenting styles are associated with internalizing and externalizing behavior. European Child on Adolescentce Psyquiatric, 21, 79-85.

Buestan, J., T. Mendieta., 2012. Practicas de crianza y su influencia en el desarrollo integral de niños de 0 a 5 años. Tesis de Licenciatura, Universidad de Cuenca, Ecuador, 69 pp.

Burchinal, M.R., E. Peisner-Feinberg, R. Pianta, C. Howes, 2002. Development of academic skills from preschool through second grade: Family and classroom predictors of developmental trajectories, Journal of School Psychology, 40, 415-436.

Ceballos, E., M.J. Rodrigo, 1998. Las metas y estrategias de socialización entre padres e hijos. En: Rodrigo, M.J., J. Palacios, Familia y desarrollo humano (págs. 225-243). Madrid: Alianza Editorial.

Chang, L., 2003. Variable effects of children's aggression, social withdrawal, and prosocial leadership as functions of teacher beliefs and behaviors. Child Development, 74, 538-548.

Chao, R.K., 1994. Beyond parental control and authoritarian parenting style: Understanding Chinese parenting through the cultural notion of training. Child Development, 65(1), 111-119.

Clereci, G., M. García, 2010. Autoconcepto y percepción de pautas de crianza en niños escolares. Anuario de investigaciones, 17, 205-2012.

Coloma, J., 1993. Estilos educativos paternos. Madrid: Narcea S.A.

Coronel, M.A., F.P. Sanchéz, 2013. Estilos de cuidado-crianza en niños y niñas de 0 a 2 años de zonas urbanas y rurales de la ciudad de Cuenca. Tesis de Licenciatura, Universidad de Cuenca, Ecuador, $151 \mathrm{pp}$.

Corvo, K., K. Williams, 2000. Substance abuse, parenting styles and agression: an exploratory study of weapon carrying students, Education, 46 (1), 1-13.

Elzo, J., 2010. Una tipología de los españoles 2008, atendiendo a sus sistemas de valores. En: Elzo, J., M. Silvestre (Eds.). Un individualismo placentero y protegido: cuarta encuesta europea de valores en su aplicación a España, pp. 251-298. Bilbao: Universidad de Deusto.

Estébez, E., S. Murgui, D. Moreno, G. Musitu, 2007. Estilos de comunicación familiar, actitud hacia la autoridad institucional y conducta violenta del adolescente en la escuela. Psichothema, 19, 108-113.

García, M., C.S. Pelegrina, J. Lendínez, 2002. Los estilos educativos de los padres y la competencia psicosocial de los adolescentes. Anuario de Psicología, 33(1), 79-95.

García, F., E. Gracia, 2010. ¿Qué estilo de socialización parental es el idóneo en España? Un estudio con niños y adolescentes de 10 a 14 años. Infancia y Aprendizaje, 33(3), 365-384.

García-Linares, M., M. Torre, M. Carpio, M. Cerezo, P. Casanova, 2014. Consistencia/ Inconsistencia en los estilos educativos de padres y madres, y estrés cotidiano en la adolescencia. Revista de Psicodidáctica, 19(2), 307-325.

Gómez, O., R. Del Rey, J. Casas, R. Ortega, 2014. Estilos parentales y variables e implicación en bullying. Cultura y Educación, 26(1), 132-158.

Gracia, C.R., M.D. Sammel, E.W. Freeman, L. Liu, L. Hollander, D.B. Nelson, 2004. Predictors of decreased libido in women during the late reproductive years. Menopause, 11(2), 144-150.

Grusec, J.E., 2002. Parental socialization and children's acquisition of values. En: Bornstein, M.H. (Ed.). Handbook of Parenting: Vol. 5. Practical issues in parenting, pp. 143-167. Mahwah, N.J.: Erlbaum.

Grusec, J.E., J.J. Goodnow, 1994. Impact of parental discipline methods on the child's internalization of values: A reconceptualization of current points of view. Development Psychology, 30, 4-19. 
Guerra, M., 2009. Estilos parentales y su influencia en el desarrollo cognitivo de preescolares de 5 a 6 años. Tesis de maestría sin publicar, Universidad Tecnológica Equinoccial, Quito, Ecuador.

Henao, G., C. Ramírez, L. Ramírez, 2007. Las practicas educativas familiares como facilitadoras del proceso de desarrollo en el niño y niña. Psicothema, 17(1), 76-82.

Hernando, A., A. Olivia, M.A. Pertegal, 2012. Variables familiares y rendimiento académico en la adolescencia. Estudios de Psicología, 33(1), 51-65.

Herrera, E., A.G. Brito, J. Pérez, M.T. Martínez, A. Díaz, 2001. Percepción de estilos educativos parentales e inadaptación en adolescentes, Revista de Psicología Universitas Tarraconensis, 23(1-2), 44-57.

Hoffman, M.L., 1975a. Moral internalization, parent power, and the nature of parent-child interaction, Developmental Psychology, 11, 228-239.

Hoffman, M.L., 1975b. Altruistic behavior and the parent-child relationship, Journal of Personality and Social Development, 31, 937-943.

Izzedin, R., A. Pachajoa, 2009. Pautas, practicas y creeencias acerca de la crianza ... ayer y hoy. Scielo, 15(2), 109-115.

Jiménez, D., Y. Guevara, 2010. Estilos de crianza e interacciones familiares: aproximaciones teóricas e investigaciones empíricas. Red Mexicana de Investigación en Psicología Educativa Sistema Mexicano de Investigación en Psicología. Tendencias en Psicología y Educación, 1, 11-41.

Kim,H., G. Chung, 2003. Relationship of recalled parenting style to self-perception in KoreanAmerican college students. Journal of genetic psychology, 164(4), 481-492.

Kim, K., R.P. Rohner, 2002. Parental warmth, control and involvement in schooling. Predicting academic achievement among Korean American adolescents, Journal of Crosscultural Psychology, 33(2), 127-140.

Lamborn, S.D., N.S. Mounts, L. Steinberg, S.M. Dornbusch, 1991. Patterns of competence and adjustment among adolescents from authoritative, authoritarian, indulgent and neglectful families, Child Development, 62(5), 1049-1065.

Llanova, L., F. Méndez, 2012. Manual del psicologo familiar. Un nuevo perfil profesional. Madrid: Piramide.

Maccoby, E.E., J.A. Martín, 1983. Socialization in the context of the family: parent-child interaction. In: Hetherington, E.M., P.H. Mussen (Eds.). Handbook of child psychology: Vol. 4. Socialization, personality and social development, pp.1-101. New York: Wiley.

Martínez, L., A. Fuertes,1999. Importancia del clima familiar y la experiencia de pareja en las relaciones de amistad de los adolescentes, Revista de Psicología Social, 14, 235-250.

Meil, G., 2006. Padres e hijos en la España actual . Barcelona: Fundación La Caixa.

Mestre, V., D. Frías,1997 Estilos educativos paternos y desarrollo del autoconcepto en los hijos, Surgam, 449, 17-35.

Molpereces, M.A., I. Llinares, G. Musitu, 2000. Estilos de disciplina familiar y prioridades de valor en la adolescencia. Revista de Psicología Social Aplicada, 11(3), 49-67.

Molpereces, M.A., G. Musitu, S. Lila, 1994. La socialización del sistema de valores en el ámbito familiar. En: Musitu, G., P. Allat (Eds.). Psicosociología de la familia, pp. 121-146. Valencia: Albatros.

Millon, T., 2000. Desarrollo de la personalidad: origen, secuencia y evolución. Trastornos de la personalidad. Mas allá del DSM-IV, 85-139. Barcelona: Millon\&R.D.DAvis.

Mínguez, R., 2012. Ética de la vida familiar y transmisión de valores morales. Universidad de Murcia. Facultad de Educación, Murcia, España.

Muñoz, A., 2005. La familia como contexto de desarrollo infantil. Dimensiones de análisis relevantes para la intervención educativa y social. Portularia, 5(2), 147-163.

Musitu, G., M.J. Cava, 2001. La familia y la educación (1a ed.). Barcelona: Octaedro. 
Musitu, G., F. García, 2004. ESPA29: Escala de estilos de socialización parental en la adolescencia. Madrid: TEA.

Musitu, G., T. Jiménez, A. Povedado, 2009. Familia y Escuela: escenarios de riesgo y protección en la violencia escolar. Revista Electrónica de Motivación y Emoción, XII (32-33), 1-26.

Musitu, G., J.M. Román, M. Gutiérrez, 1996. Educación familiar y socialización de los hijos. Barcelona: Idea Books.

Observatorio de los Derechos de la Niñez y la Adolescencia, 2010. Los niños y las niñas del Ecuador a inicios del siglo XXI. Quito: Noción.

Olivia, A., A. Parra, 2004. Contexto familiar y desarrollo psicológico durante la adolescencia. En: Arranz E. (Ed.). Familia y Desarrollo psicológico, pp. 96-123. Madrid: Pearson Prentice Hall.

Olivia, A., A. Parra, E. Arranz, 2008. Estilos relacionales parentales y ajustes adolescentes. Infancia y Aprendizaje, 31, 93-106.

Orte Socias, C., 2005. Los programas de prevención de drogas centrados en la familia. Una visión desde la investigación práctica: Proyecto Hombre. Revista de la Asociación Proyecto Hombre, 53, 14-17.

Orte Socias, C., 2006. Volver a ser una familia después de la droga. Aplicación de un programa para reforzar la competencia familiar: Proyecto Hombre. Revista de la Asociación Proyecto Hombre, 57, 43-46.

Ortega, P., R. Mínguez, 2003. Familia y transmisión de valores. Teoría de la Educación. Revista Interuniversitaria, 15, 33-56.

Palacios, J., 1999. La familia y su papel en el desarrollo afectivo y social. En: López, F., I. Etxebarría, M.J. Ortiz (Eds.). Desarrollo afectivo y social, pp. 267-284. Madrid: Pirámide.

Palacios, J., M.C. Moreno, 1995. Contexto familiar y desarrollo social. En: Rodrigo, M.J. (Ed.). Contexto y desarrollo social, pp. 157-188. Madrid: Síntesis Psicología.

Palacios, J., M.J. Rodrigo, 1998. La familia como contexto de desarrollo. En: Rodrigo, M.J., J. Palacios. Familia y desarrollo humano, pp. 225-243. Madrid: Alianza Editorial.

Peralbo, M., M.L. Fernández, 2003. Estructura familiar y rendimiento escolar en educación secundaria obligatoria. Revista Galego-Portuguesa de Psicoloxía e Educación, 7 (8), 309-322.

Pérez, P., P.M. Vidal, M., 2001. Valores y pautas de la crianza familiar en los montes del Pas. Teoría de la Educación: Revista Interuniversitaria, 13, 115-136.

Perez, P., 2012. La socialización parental en padres españoles con hijos de 6 a 14 años. Psicothema, 24(3), 371-376.

Pons-Salvador, G., M. Cerezo, G. Bernabé, 2005. Cambio y estabilidad en los factores que afectan negativamente a la parentalidad. Psicothema, 17(1), 31-36.

Ramírez, M.A., 2005. Padres y desarrollo de los hijos. Prácticas de crianza. Estudios Pedagógicos XXXI(2), 167-177.

Raya, A., 2008. Estudio sobre los estilos educativos parentales y su relación con los trastornos de conducta en la infancia. Córdoba, España: Servicio de Publicaciones de la Universidad de Córdoba.

Rich Harris, J., 2002. El mito de la educación. Barcelona: Randon House Mondadory.

Rodrigo, M.J., J. Palacios, 1998. Conceptos y dimensiones en el análisis evolutivo-educativo de la familia. En: Rodrigo, M., J. Palacios (coord.). Familia y Desarrollo Humano, pp. 45-70.

Rodrigo, M.J., M.L.Máiquez, M. García, R. Mendoza, A. Rubio, A. Martínez, J.C. Martín, 2004. Relaciones padres-hijos y estilos de vida en la adolescencia, Psicothema, 16(2), 203-210.

Rodríguez, M.A., M.V. Del barrio, M.A. Carrasco, 2009. ¿Como perciben los hijos la crianza materna y paterna? Diferencias por edad y sexo. Escritos de Psicología, 2(2), 10-18.

Rodríguez, M.A., M.V. Del barrio, M.A. Carrasco, 2009.Consistencia Interparental y su relación con la agresión y la sintomatología depresiva en niños y adolescentes. Revista de Psicopatología y Psicología Clínica, 14(1), 51-60. 
Sandoval, J., 2006. Ambiente escolar, familiar y comunitario en relación con los compotamientos agresivos y prosociales en niños de 3 a 12 años. Rev. Fac. Nac. Salud Pública, 24(1), 30-39.

Shephard, B., M. Ordóñez, C. Mora, 2015. Estudio Descriptivo: Programa de prevención y disminución del acoso escolar - "Bullying". Fase Diagnóstica: Prevalencia. Rev. Med. HJCA, $7(2), 155-161$.

Torío, S., 2003. Estudio socioeducativo de hábitos y tendencias de comportamiento en familias con niños de educación infantil y primaria en Asturias. Oviedo, España: Universidad de Oviedo, Servicio de Publicaciones.

Torío, S., J.V. Peña, M. Inda, 2008a. Estilos de educación familiar. Psicothema, 20(1), 62-70.

Torio, S., J.V. Peña, M. del Carmen Rodriguez, 2008b. Estilos educativos parentales, Revisión bibliográfica y reformulación teórica. Teoría de la Educación, 20, 151-178. 